\title{
Constructing Core Backbone Grid Based on the Index System of Power Grid Survivability and BBO Algorithm
}

\author{
Feifei Dong, Dichen Liu, Jun Wu, Fei Tang, Chunli Song, Haolei Wang, Lina Ke, \\ Bingcheng Cen, Wentao Sun, and Zhenshan Zhu
}

\author{
School of Electric Engineering, Wuhan University, Wuhan 430072, China \\ Correspondence should be addressed to Jun Wu; flysky007dong@163.com
}

Received 18 January 2014; Revised 4 May 2014; Accepted 19 May 2014; Published 5 June 2014

Academic Editor: Xinghuo Yu

Copyright (c) 2014 Feifei Dong et al. This is an open access article distributed under the Creative Commons Attribution License, which permits unrestricted use, distribution, and reproduction in any medium, provided the original work is properly cited.

\begin{abstract}
Constructing core backbone grid is conducive to strengthen the construction of grid structure and improve the ability of withstanding natural disasters. The survivability index of power grid is made up of four indices, namely, the resistibility, recoverability, security, and connectivity. Based on survivability, a method of constructing core backbone grid with the optimal criteria of the minimal total line length and the largest integrated survivability index is put forward. The biogeography-based optimization (BBO) algorithm is used to search for the core backbone grid. Compared with the traditional algorithms, $\mathrm{BBO}$ algorithm shows advantages in fast speed of convergence and high convergence precision. Moreover, the searching results for three kinds of objective functions by $\mathrm{BBO}$ algorithm verify the effectiveness of the proposed model of constructing core backbone grid.
\end{abstract}

\section{Introduction}

For the past few years, power grid in China is constantly damaged by extreme natural disasters due to the failure of past standards of power facilities unable to resist the increasing natural disasters $[1,2]$. Therefore, it is necessary to design the standards of disaster resistance levels differentially, according to the various lines' geographical landforms and climate conditions where power transmission lines are crossed. The goal of differential planning design is to confirm the core backbone grid, which is made up of important lines that can guarantee continuous power supply of the important load when the major natural disasters attack $[3,4]$. Constructing core backbone grid is significant to improve the stability of the power grid's structure, reduce the secondary investment of repairing, and rebuild the harm of power grid caused by natural disasters, as well as guarantee power grid's safe and reliable operation under severe natural disasters $[5,6]$.

The concept of survivability is firstly put forward by Hollway and Neumann [7] in 1993. Survivability of system refers to the ability that the system can complete its critical services in a timely manner and restore its basic services as soon as possible when it is subjected to an attack, failure, or a sudden incident [8]. Survivability has been widely concerned in the complex network and information system as a new research area $[9,10]$, but its application in the related fields of power system is relatively less $[11,12]$. Considering survivability is helpful to keep the system alive with supportive network structure. In that case, the constructed core backbone grid has a strong resistance, restorative ability, and connectivity. A key method of line identification based on network survivability evaluation was proposed in [13]. A searching model and a searching method of backbone grid were also presented, which has certain enlightening significance. But the survivability index of this method is relatively simple, and the search algorithm is easy to fall into local optimal solution. The survivability index system that can systematically reflect information system is proposed in [14]. The formalized description and mathematical model were given as well. It has done pioneered work in establishing survivability index of power system.

The search of core backbone grid involves nonlinear, multivariate, and discontinuous optimization problem, which depends on artificial intelligence algorithms $[15,16]$. As a new kind of artificial intelligence algorithm, BBO algorithm based on species migration patterns has achieved good results in 
parameter identification [17], fault diagnosis [18], and image classification [19], as well as transmission network planning [20] and other fields [21,22]. It is shown that the algorithm has advantages of less set parameters, light computational work, fast convergence speed, and good stability. Therefore, BBO algorithm is adopted to search the optimal solution of core backbone grid in this paper.

A new method of constructing core backbone grid considering survivability is put forward in this paper. The indices of survivability are built from factors of resistibility, recoverability, security, and connectivity. The largest integrated survivability index and the minimum total length of the backbone grid's lines are regarded as the optimal solution of objective function, given network connectivity and power grid's safe operation as constraint conditions. BBO algorithm provided with strong search ability is used to search backbone grid. Empirical simulations show that the proposed method is accurate and effective, with additional advantages of fast convergence speed and high convergence precision over the methods such as particle swarm optimization (PSO), binary ant colony algorithm (BACA), and genetic algorithm (GA).

\section{Basic Method of Constructing Core Backbone Grid}

The key of differential planning design is to determine the selecting principles and construction standards of affordable power supply, load, and network frame. Then, the quantitative evaluation method is adopted to identify the key components and build core backbone grid, increasing the resistant standards of disasters of element differentially. In this way, the system guarantees the delivery capacity of important load and power supply as well as the ability of the interval exchange capacity, promising the system recovered and reconstructed on the basis of the core backbone grid. The core method lies in that it is the viability and self-healing ability instead of the emergency control and scheduling dispatch during disaster and the repair and reconstruction after disaster that alleviate the damage of major natural disasters to the power grid. Therefore, the core backbone grid needs to satisfy the following conditions: (a) to guarantee the continuous power supply of the important load and the reliable transmission of the important power; (b) to make the rack satisfy the constraints of power grid's safe operation as well as network's topology connectivity; (c) to meet the total length of the subrack's line to be shortest from the perspective of economy; (d) to meet the survivability of the subrack to be stronger from the perspective of the optimal network topology configuration.

Survivability is one of the most important constraints of the core backbone grid, and it has great influence in resistance ability, resilience, and the stability of the overall network topology of the rack against natural disaster. Therefore, establishing an index system of survivability effectively and then determining the comprehensive indicators of network survivability are the key steps to construct core backbone grid.

\section{The Index System of Power Grid Survivability}

Survivability of power grid is defined as the ability of ensuring continuous power supply to important loads that relied on the high design standards of the core backbone grid, as well as restoring power supply for other loads gradually based on the network frame when major natural disasters attack. The index system of survivability is built from these four aspects as follows: resistibility, recoverability, security, and connectivity, by which the integrated index of survivability is finally obtained.

3.1. The Index of Resistibility. Resistibility of power grid reflects the resistance where the system provides power supply for the important loads with major natural disasters. The three indicators, namely, preserving rate of lines, preserving rate of nodes, and preserving rate of loads, are introduced to evaluate resistibility.

Preserving rate of lines $\alpha_{l}$ is as follows:

$$
\alpha_{l}=\frac{\operatorname{dim}(L)-L \_ \text {failure }}{\operatorname{dim}(L)},
$$

where $\operatorname{dim}(L)$ is the number of original rack's lines and $L_{-}$failure is the number of failure lines of the remaining network frame after natural disasters compared to the original rack.

Preserving rate of nodes $\alpha_{b}$ is as follows:

$$
\alpha_{b}=\frac{\operatorname{dim}(B)-B \_ \text {failure }}{\operatorname{dim}(B)},
$$

where $\operatorname{dim}(B)$ is the number of original rack's nodes and $B$ failure is the number of failure nodes of the remaining network frame after natural disasters compared to the original rack.

Preserving rate of loads $\alpha_{c}$ is as follows:

$$
\alpha_{c}=\frac{\operatorname{dim}(C)-C_{\text {ffailure }}}{\operatorname{dim}(C)},
$$

where $\operatorname{dim}(C)$ is the number of original rack's lodes and $C$ failure is the number of failure loads of the remaining network frame after natural disasters compared to the original rack.

3.2. The Index of Recoverability. Recoverability of power grid reflects whether the power grid can recover after suffering from natural disasters, and to what extent it can recover. The generator standby indicator and load recovery degree index are introduced to evaluate recoverability.

The generator standby indicator $\beta_{g}$ is as follows:

$$
\beta_{g}=\sum_{i=1}^{m_{g}}\left(\frac{G_{i \max }-G_{i}}{G_{i \max }}\right),
$$

where $G_{i}$ is the actual output of the generator $i$ in the backbone grid, which is the output power of the generator $i$ obtained by power flow calculation of the core backbone grid. $G_{i \max }$ is the largest capacity of the generator $i$ in the backbone grid. $m_{g}$ is the total number of generators. 
The load recovery degree index $\beta_{c}$ is as follows:

$$
\beta_{c}=\frac{\sum_{j=1}^{\operatorname{dim}(B)-B_{-} \text {failure }} S_{j m}-S_{j}}{\sum_{j=1}^{\operatorname{dim}(B)-B_{-} \text {failure }} S_{j m}},
$$

where $S_{j m}$ is the active load of node $j$ in the backbone network frame scheme, which is the biggest active load that node $j$ can bear. $S_{j}$ is the actual active load of node $j$ in the backbone network frame scheme, which is the active load that node $j$ needs to afford according to the scheme of the backbone grid. They need to meet the safe operation conditions and ensure the rack's load to be largest.

3.3. The Index of Security. The less important lines of core backbone grid after natural disasters are checked by $N-1$ criteria; the safety margin of core backbone grid is analyzed by the index of security called $S f t$, mainly including the index of bus voltage fluctuation and index of branch power fluctuation.

The difference between the current bus voltage and upper or lower limits of bus voltage is regarded as the index of bus voltage fluctuation, namely, $\gamma_{L j}$, which is to measure the current bus voltage with following expression:

$$
\gamma_{L j}= \begin{cases}\frac{\left|U_{j}\right|-\left|U_{j 0}\right|}{\left|U_{j, \max }\right|-\left|U_{j 0}\right|} & \left|U_{j}\right|>\left|U_{j 0}\right| \\ \frac{\left|U_{j 0}\right|-\left|U_{j}\right|}{\left|U_{j 0}\right|-\left|U_{j, \min }\right|} & \left|U_{j}\right|<\left|U_{j 0}\right|,\end{cases}
$$

where $\left|U_{j}\right|,\left|U_{j 0}\right|,\left|U_{j, \text { max }}\right|$, and $\left|U_{j, \text { min }}\right|$ are the amplitude of current voltage, nominal amplitude, the upper limit, and the lower limit of Bus $j$, respectively.

The bus voltage fluctuations of all nodes in the core backbone grid are taken advantages as the average bus voltage fluctuation, namely, $\gamma_{L}$, which can be expressed as

$$
\gamma_{L}=\frac{1}{\operatorname{dim}(B)-B_{-} \text {failure }} \sum_{j=1}^{\operatorname{dim}(B)-B_{-} \text {failure }} \gamma_{L j} .
$$

The difference between the current branch power and upper or lower limit of branch power is regarded as the index of branch power fluctuation, namely, $\lambda_{L k}$, which is used to analyze the current branch power flow. Its expression is as follows:

$$
\lambda_{L k}= \begin{cases}\frac{\left|P_{k}\right|-\left|P_{k 0}\right|}{\left|P_{k, \max }\right|-\left|P_{k 0}\right|} & \left|P_{k}\right|>\left|P_{k 0}\right| \\ \frac{\left|P_{k 0}\right|-\left|P_{k}\right|}{\left|P_{k 0}\right|-\left|P_{k, \text { min }}\right|} & \left|P_{k}\right|<\left|P_{k 0}\right|,\end{cases}
$$

where $\left|P_{k}\right|,\left|P_{k 0}\right|,\left|P_{k, \max }\right|$, and $\left|P_{k, \text { min }}\right|$ are the amplitude of current power, nominal amplitude, the upper limit, and the lower limit of Branch $k$, respectively.

The average branch power fluctuations, $\lambda_{L}$, are defined as the mean of the sum of all lines of the branch power fluctuations in the core backbone grid with the expression of

$$
\lambda_{L}=\frac{1}{\operatorname{dim}(L)-L \_ \text {failure }} \sum_{k=1}^{\operatorname{dim}(L)-L_{\text {failure }}} \lambda_{L k} .
$$

3.4. The Index of Connectivity. The relative tightness degree and the relative condensation degree of the grid are introduced to evaluate connectivity.

(1) The Relative Tightness Degree of the Grid. If the node $j$ has $K_{j}$ adjacent nodes, there are at most $K_{j}\left(K_{j}-1\right) / 2$ lines among these nodes. Assuming that the actual linking lines are $t_{j}$, in that case, the clustering coefficient of the node $j$ is defined as follows:

$$
C_{j}=\frac{2 t_{j}}{K_{j}\left(K_{j}-1\right)} .
$$

The tightness degree of the grid is obtained by the weighted average of all nodes of $C_{j}$ in backbone grid, which is a parameter that shows the connected degree of neighboring nodes, and is expressed as

$$
\begin{aligned}
C & =\frac{\sum_{j=1}^{\operatorname{dim}(B)-B_{-} \text {failure }} C_{j}}{\operatorname{dim}(B)-B_{-} \text {failure }} \\
& =\frac{\sum_{j=1}^{\operatorname{dim}(B)-B_{\text {failure }}} 2 t_{j} / K_{j}\left(K_{j}-1\right)}{\operatorname{dim}(B)-B_{\text {failure }}} .
\end{aligned}
$$

If the tightness degree of the original grid is $C_{0}$, the relative tightness degree of the backbone grid is as follows:

$$
\varphi=\frac{C}{C_{0}} .
$$

(2) The Relative Condensation Degree of the Grid. The relative condensation degree of the grid is the reciprocal of the product of the number of nodes and the weighted average of the shortest path shown as

$$
\partial=\frac{1}{n \cdot l} .
$$

The traditional shortest path is the minimum number of edges between two nodes. Considering the actual conditions of power system and assigning the length of the transmission line as the lines' weight coefficients, the weighted average of the shortest path of backbone grid is as follows:

$$
l=\frac{2}{n \times(n-1)} \sum d_{i j}^{\prime}
$$

where $d_{i j}^{\prime}$ is the weighted number of edges that the shortest path passes through and $n$ is the total number of nodes in the grid.

By substituting formula (14) into formula (13), the condensation degree of the backbone grid is

$$
\partial=\frac{n-1}{2 \sum d_{i j}^{\prime}}=\frac{\operatorname{dim}(B)-B_{-} \text {failure }-1}{2 \sum d_{i j}^{\prime}} .
$$

Given the condensation degree of the original grid as $\partial_{0}$, the relative condensation degree of the backbone grid is as follows:

$$
\phi=\frac{\partial}{\partial_{0}}
$$


3.5. Integrated Index of Survivability. There are four indicators included in the index system of power grid survivability. Primary level (Level 1) of index is the integrated index of survivability, while the secondary level (Level 2) of indices is resistibility, recoverability, security, and connectivity index, and the tertiary level (Level 3) of indices is made up by refining indicators of each secondary index. The indexes of Level $m$ can be calculated by the indexes of Level $m+1$ according to the following principles.

Vector $\mathbf{R}_{m}=\left(R_{m 1}, R_{m 2}, \ldots, R_{m \eta}\right)^{T}$ is set as the individual risk index vector of survivability's Level $m$ indexes. $\eta$ is the total number of Level $m+1$ indicators. $R_{m i}$ is the single index $i$ of Level $m$ indexes. The survivability index of Level $m$ is defined as follows:

$$
\mathbf{R}_{m s}=a_{1} \times \frac{1}{\eta}\left\|\mathbf{R}_{m}\right\|_{1}+b_{1} \times\left\|\mathbf{R}_{m}\right\|_{\infty},
$$

where $a_{1}$ and $b_{1}$ are weight coefficients to satisfy the equation of $a_{1}+b_{1}=1$ and $\left\|\mathbf{R}_{m}\right\|_{1}$ and $\left\|\mathbf{R}_{m}\right\|_{\infty}$ are 1 norm and $\infty$ norm of vector $\mathbf{R}_{m}$, respectively. Consider

$$
\begin{aligned}
\left\|\mathbf{R}_{m}\right\|_{1} & =\sum_{i=1}^{\eta}\left|R_{m i}\right|, \\
\left\|\mathbf{R}_{m}\right\|_{\infty} & =\max \left|R_{m i}\right| .
\end{aligned}
$$

Therefore, the integrated index of survivability is expressed as follows:

$$
\operatorname{Surv}=\alpha \times \frac{1}{4}\|\mathbf{R}\|_{1}+\beta \times\|\mathbf{R}\|_{\infty},
$$

where $\alpha$ and $\beta$ are weighted coefficients that meet the need of $\alpha+\beta=1 ; \mathbf{R}=(\text { Resis, Recov, Sft }, \text { Connec })^{T}$.

\section{The Mathematical Model of Constructing the Core Backbone Grid}

The core backbone grid includes (1) the important power source nodes; (2) load nodes; (3) key circuits; (4) the nodes connected to the key circuits that should be guaranteed according to the practical situation; (5) the lines and nodes that need to be preserved considering the constrains of network connectivity, tide of constraints, the shortest length of total lines, and the largest integrated index of survivability. Therefore, mathematical model of constructing core backbone grid is as follows:

$$
\begin{aligned}
& \min \quad f=\frac{L_{1} / L_{0}}{\operatorname{Surv}} \\
& \text { s.t. } \quad L_{1}=\sum_{i=1}^{\operatorname{dim}(L)-L_{-} \text {failure }} l_{i} x_{i} \\
& L_{0}=\sum_{i=1}^{\operatorname{dim}(L)} l_{i} x_{i} \\
& \phi(x)=\phi\left(x_{1}, x_{2}, \ldots, x_{l}\right)=1
\end{aligned}
$$

$$
\begin{aligned}
& g(x)=0 \\
& h(x) \leq 0 .
\end{aligned}
$$

In the formula, $x_{i}$ denotes the on or off state of the lines; 1 means on and 0 means off. $l_{i}$ is the length of line $i . L_{1}$ is the total length of the backbone grid and $L_{0}$ is that of the original grid. $L_{1} / L_{0}$ is the normalized value of the backbone grid's total length compared with the original grid. The value of $L_{1} / L_{0}$ is in the $(0,1)$ and compared with Surv. $\phi(x)$ is the function to test the connectivity. $\phi(x)=1$ refers to the fact that the network is connected, while $\phi(x)=0$ indicates that the network is disconnected. $g(x)=0$ is the equality constraint for the power flow. $h(x) \leq 0$ is the inequality constraint for the power flow.

\section{Search of the Backbone Grid Based on BBO Algorithm}

5.1. The Basic Method of BBO Algorithm. Biogeographybased optimization algorithm is a new type of evolutionary algorithm that uses the biogeography theory to solve optimization problems. Its basic idea is as follows. Firstly, a number of relatively independent habitats are constructed as candidate solutions. Secondly, the species share information through migration between habitats and update the information through mutation. Finally, the most fitted habitat is selected, and the optimal solution of the problem is obtained $[23,24]$. For the multiobjective optimization problems of the core backbone grid, the appropriate index HIS is adopted to express the objective function of the mathematical model of constructing core backbone grid, that is, $\left(L_{1} / L_{0}\right) /$ Surv. The variable SIV is used to show variables included in each habitat, namely, the state of lines. There are mainly two operations included in the algorithm, migration and mutation.

(1) Migration Operation. The migration operation is for the information exchange with other habitats, in order to search for the solution in the wide area. Since the linear species migration model cannot accurately simulate the complex process of species migration of the practical biological environment, the cosine migration model which is more similar to the nature process is adopted to simulate migration rate and is shown in Figure 1.

The immigration rate $\lambda(S)$ and emigration rate $\mu(S)$ of cosine migration model are shown as follows, respectively:

$$
\begin{aligned}
& \lambda(S)=\frac{I}{2}\left(\cos \left(\frac{S \pi}{S_{m}}\right)+1\right), \\
& \mu(S)=\frac{E}{2}\left(-\cos \left(\frac{S \pi}{S_{m}}\right)+1\right) .
\end{aligned}
$$

In this migration model, $I$ denotes the largest immigration rate, $E$ is the biggest emigration rate, $S$ is the number of species, and $S_{m}$ is the maximum number of species. When there are less or more species in the habitat, immigration rate and emigration rate change smoothly. When habitat has a certain number of species, immigration rate and emigration rate change relatively fast. 


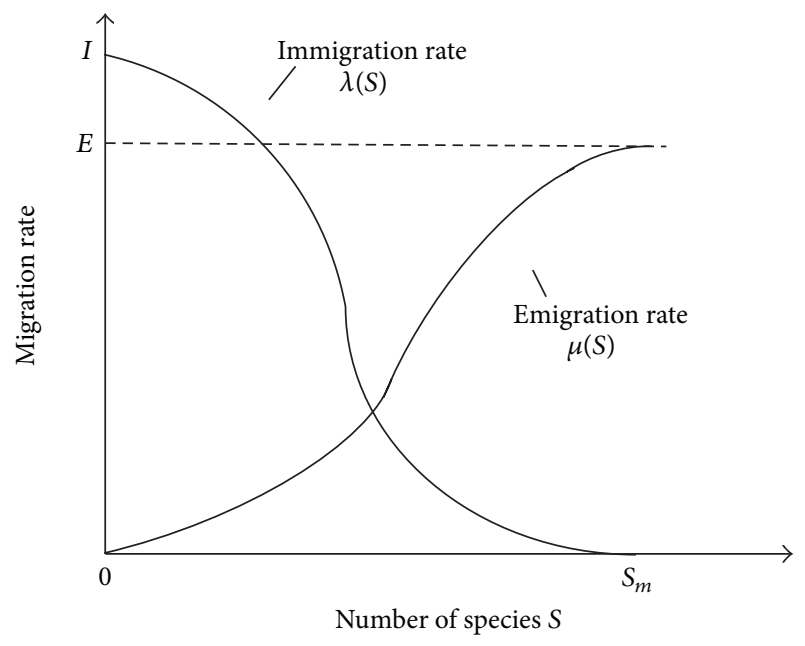

Figure 1: Cosine migration model.

(2) Mutation Operation. Mutation operation is to increase the diversity of population. The probability of the habitat, $P_{s}$, has a number of species $S$. The variation rate is found out through the following formula:

$$
M(\mathrm{~S})=M_{\max }\left(1-\frac{P_{s}}{P_{m}}\right),
$$

where $M_{\max }$ is the biggest mutation rate and it can be adjusted according to the requirements of different users. $P_{m}$ is the maximum of $P_{s}$.

Mutation operation makes the solution of lower HIS improve by variation and makes the higher HIS obtain the opportunity of improving their solutions.

\subsection{Search of Core Backbone Grid Based on BBO Algorithm.} The multiobjective optimization problem of constructing the core backbone grid is solved by $\mathrm{BBO}$ algorithm to obtain the high survivability. Specific steps are as follows.

Step 1. Initiate the original grid's parameters needed, including the node loads, the output of generators, and the proposed paths. The control parameters of the BBO algorithm are initialized as well. The initial population $P$ that meets the constraint condition is randomly generated.

Step 2. The suitability index of the habitat is calculated and sorted. The individual optimal solution, $X_{\text {best }}$, is saved and then judged whether it meets the end condition. If yes, the results are output and then converted to backbone grid scheme and, in that case, the program is over. Otherwise, continue to Step 3.

Step 3. The cosine migration model is established. The habitat's species number, immigration rate, and emigration rate are calculated.

Step 4. The migration operation is performed to form a new population $P 1$. The suitability index of the habitat is recalculated, and then the optimal solution $X_{\text {best } 1}$ is updated.

Step 5. The mutation operation is carried out, and then the optimal solution of the population $X_{\text {best } 2}$ is updated.

Step 6. Determine whether it meets the maximum number of iterations. If satisfied, the results are output and then converted to core backbone grid scheme. In that case, the program is over. Otherwise, go to Step 2.

\section{Case Study}

In order to verify the effectiveness of the proposed method, the core backbone grid of IEEE118 node system is constructed based on $\mathrm{BBO}$ algorithm, taking the power grid's survivability into account. The system includes 118 nodes and 179 branches, with 53 generator nodes. Except for the nodes of 17, 22, 23, 57, $58,84,102,108,109$, and 114, other load nodes are all important loads.

The parameters of $\mathrm{BBO}$ algorithm are set as follows: the population size $N$ is set as 50 , the largest number of iterations $k_{\max }$ is 200 , the largest mutation rate $M_{\max }$ is 0.01 , the biggest immigration rate $I$ and emigration rate $E$ are both set as 1 , and the global mobility $P_{\text {mod }}$ is 1 . The optimal scheme of core backbone grid considering survivability searched by BBO algorithm is shown in Figure 2. The solid points represent generator nodes. The hollow points denote the load nodes. The reserved lines of the core backbone grid are in the blue solid lines. This scheme of core backbone grid is composed of 71 nodes and 70 lines, including 19 generator nodes and 44 load nodes.

In order to verify the performance of $\mathrm{BBO}$ algorithm, PSO algorithm, BACA algorithm, and GA algorithm are used to search the core backbone grid, and their population size and maximum number of iterations are set the same as BBO algorithm. Based on the characteristics of randomness of the algorithm, the statistical results of the four kinds of algorithms are listed in Table 1 with total independence of 50 times. The curves of suitability index with four kinds of algorithms optimal scheme are shown in Figure 3.

From Table 1 and Figure 3, the results indicate that the best value and the worst value of $\mathrm{BBO}$ search's objective function are relatively smaller. In that case, the precision of objective function searched by $\mathrm{BBO}$ algorithm is higher. Meanwhile, BBO algorithm has got 32 searches of the optimal solution, which shows that this algorithm has a stronger convergence ability compared with other three kinds of algorithms. In addition, it can be concluded from Figure 3 that $\mathrm{BBO}$ algorithm has obvious advantages in convergence rate compared with the other three kinds of algorithms.

The optimal core backbone grid schemes of three objective functions searched by $\mathrm{BBO}$ algorithm are presented in Table 2.

Three kinds of objective functions are set as follows.

$\min f_{1}=a_{1}$ refers to the fact that the number of lines is the least, where $a_{1}$ expresses the total number of lines. 


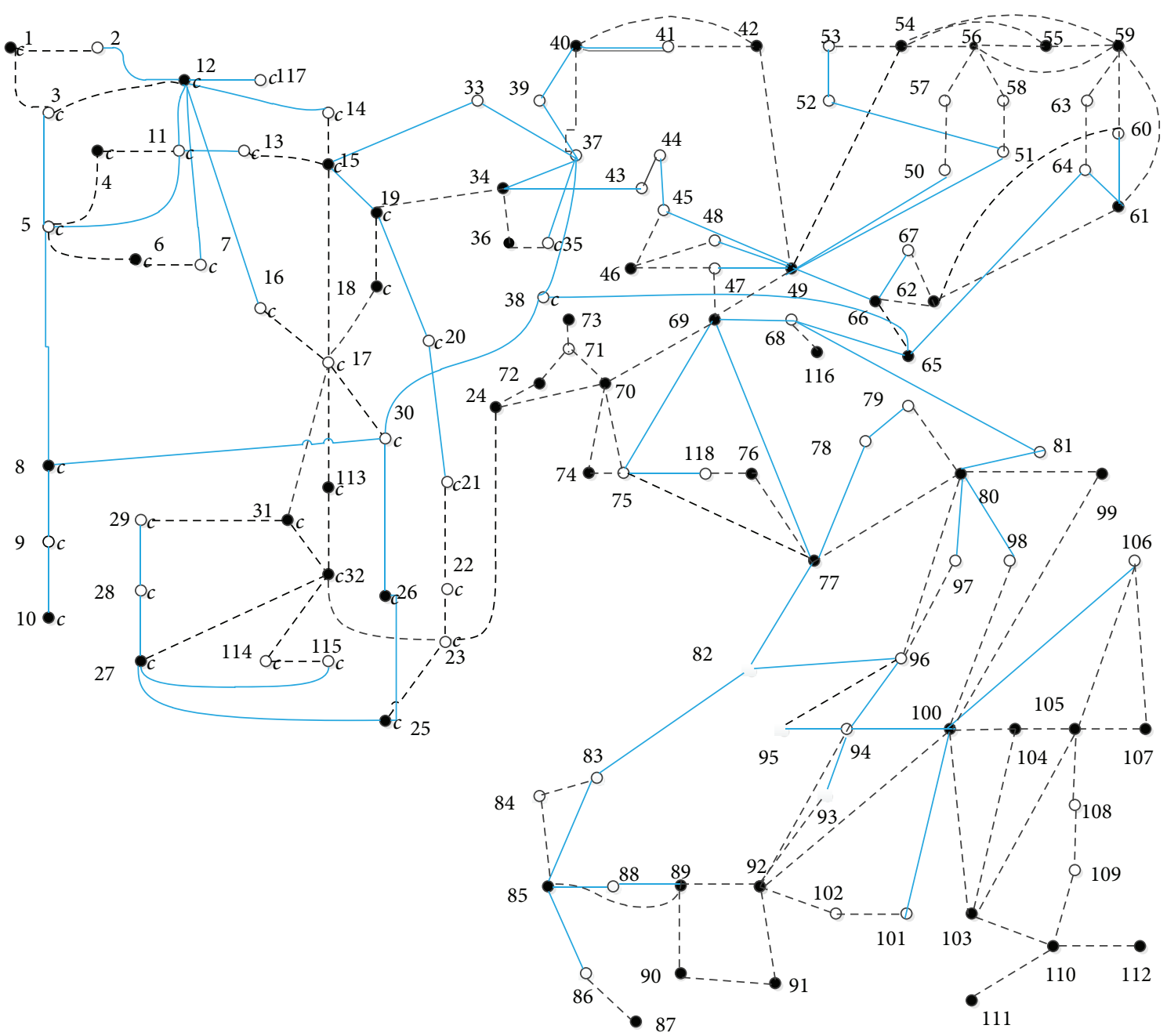

FIgURE 2: Core backbone grid scheme for IEEE 118-bus power system based on BBO algorithm.

TABLE 1: Comparison of objective function solution by different algorithms.

\begin{tabular}{lcccc}
\hline \multirow{2}{*}{ Algorithm } & \multicolumn{4}{c}{ Objective function } \\
& $\begin{array}{c}\text { Best } \\
\text { value }\end{array}$ & $\begin{array}{c}\text { Worst } \\
\text { value }\end{array}$ & $\begin{array}{c}\text { Average } \\
\text { value }\end{array}$ & $\begin{array}{c}\text { Time of optimal } \\
\text { solution }\end{array}$ \\
\hline BBO & 0.3118 & 0.3304 & 0.3163 & 32 \\
PSO & 0.3151 & 0.3433 & 0.3310 & 20 \\
BACA & 0.3252 & 0.3441 & 0.3320 & 18 \\
GA & 0.3542 & 0.3722 & 0.3610 & 25 \\
\hline
\end{tabular}

$\min f_{2}=\left(L_{1} / L_{0}\right) /$ Surv refers to the shortest length of total lines and the largest integrated survivability index.

$\min f_{3}=L_{1}$ refers to the minimum length of lines.

From Table 2, under the objective function of $\min f_{1}=$ $a_{1}$, the number of lines of the core backbone grid's optimal scheme is 67 , and the total length of lines is 6.3739. Under
TABLE 2: Comparison of the optimal scheme of core backbone grid under different objective functions based on BBO algorithm.

\begin{tabular}{lccc}
\hline Objective function & \multicolumn{3}{c}{ The optimal scheme } \\
& $a_{1}$ & $\frac{L_{1} / L_{0}}{\text { surv }}$ & $L_{1}$ \\
\hline $\min f_{1}=a_{1}$ & 67 & 0.4170 & 6.3739 \\
$\min f_{2}=\frac{L_{1} / L_{0}}{\operatorname{surv}}$ & 70 & 0.3119 & 5.4490 \\
$\min f_{3}=L_{1}$ & 75 & 0.3515 & 5.3898 \\
\hline
\end{tabular}

the objective function of $\min f_{2}=\left(L_{1} / L_{0}\right) /$ Surv, there are 70 lines in the lines' collection and the total length of lines is 5.4490. Meanwhile, 75 lines are in the core backbone grid's optimal scheme under the objective function $\min f_{3}=L_{1}$ with total length of 5.3898. Since $L_{1} / L_{0}$ in the objective function $f_{2}$ reflects the content of the objective function $f_{3}$ and the preserving rate indicators of survivability in the 


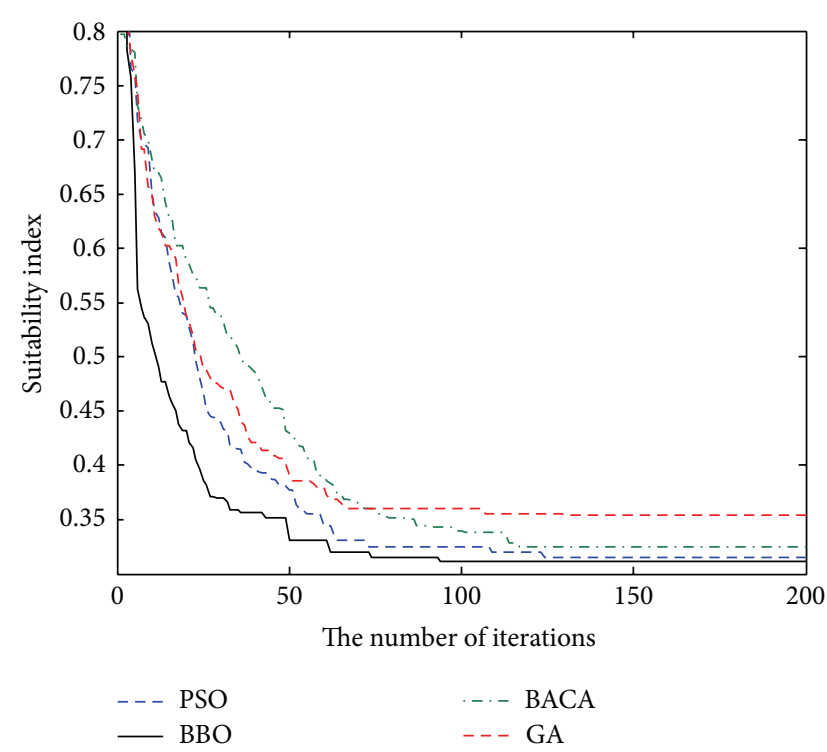

FIGURE 3: Contrast of suitability index curve of 4 kinds of algorithm optimal solution.

objective function $f_{2}$ also reflect the content of the objective function $f_{1}$, it is reasonable that the searched number of lines and the total length of lines of $f_{2}$ is between that of $f_{1}$ and $f_{3}$. Thus, the correctness and effectiveness of search algorithm proposed in this paper are verified. In addition, the objective function, $f_{2}$, of constructing the core backbone grid takes into account the resistibility, the recoverability, and the configuration of the network frame, compared with the commonly used objective functions, $f_{1}$ and $f_{3}$, which emphasizes economic benefits. This method reflects the characteristic of network frame more comprehensively and gives insights into improving the resistance of power grid against natural disasters and in differentiation design.

\section{Conclusions}

(1) The index system of survivability is proposed and is made up of resistibility, recoverability, security, and connectivity. Based on the index system of power grid survivability, the model of constructing core backbone grid with the target of the shortest length of total lines and the largest integrated survivability index is built. The results of the case study show that the model can well balance the factors of economy, resistibility, recoverability, and the configuration of the network frame giving insights of differentiated planning with reasonable design schemes.

(2) Compared with the other three kinds of traditional algorithms, $\mathrm{BBO}$ algorithm has higher precision and better convergence speed in searching for the optimal solution of core backbone grid.

(3) The system of IEEE118 nodes is studied. The multiple operational results of the four different algorithms and the search results for three kinds of objective functions by $\mathrm{BBO}$ algorithm are compared. In that case, the rationality of the proposed model and the superiority of search algorithm are verified.

\section{Nomenclature}

$\alpha_{l}$ : $\quad$ Preserving rate of line

$\operatorname{dim}(L)$ : The number of original rack's lines

$L_{-}$failure : The number of failure lines of the remaining grid

$\alpha_{b}: \quad$ Preserving rate of node

$\operatorname{dim}(B)$ : The number of original rack's nodes

$B \_$failure : The number of failure nodes of the remaining grid

$\alpha_{c}: \quad$ Preserving rate of load

$\operatorname{dim}(C)$ : The number of original rack's loads

C_failure : The number of failure loads of the remaining grid

$\beta_{g}: \quad$ Generator standby indicator

$G_{i}$ : Actual output of generator $i$ in backbone grid

$G_{i \max }$ : Largest capacity of generator $i$ in backbone grid

$\beta_{c}: \quad$ Load recovery degree index

$S_{j}$ : $\quad$ Active load of node $j$ in the backbone grid

$S_{j m}: \quad$ Actual active load of node $j$ in the backbone

$\gamma_{L j}: \quad$ Index of bus voltage fluctuation

$\left|V_{j}\right|: \quad$ The amplitude of BUS $j$ 's current voltage

$\left|V_{j 0}\right|: \quad$ Nominal amplitude of Bus $j$

$\left|V_{j, \max }\right|: \quad$ The upper limit of Bus $j$

$\left|V_{j, \min }\right|: \quad$ The lower limit of Bus $j$

$\gamma_{L}: \quad$ Average bus voltage fluctuation

$\lambda_{L k}: \quad$ Index of branch power fluctuation

$\left|P_{k}\right|$ : $\quad$ The amplitude of Branch $k$ 's current power

$\left|P_{k 0}\right|: \quad \quad \quad$ Nominal amplitude of Branch $k$

$\left|P_{k, \max }\right|:$ The upper limit of Branch $k$

$\left|P_{k, \text { min }}\right|$ : The lower limit of Branch $k$

$\lambda_{L}: \quad$ Average branch power fluctuation

$C_{j}$ : $\quad$ Clustering coefficient of the node $j$

$C$ : $\quad$ Tightness degree of the backbone grid

$C_{0}: \quad$ Tightness degree of the original grid

$\varphi$ : $\quad$ Relative tightness degree of the backbone grid

$\partial: \quad$ Condensation degree of the backbone grid

$\partial_{0}: \quad$ Condensation degree of the original grid

$\phi$ : $\quad$ Relative condensation degree of backbone grid

$x_{i}: \quad$ The state of line's input or excision

$l_{i}: \quad$ The length of line $i$

$L_{1}$ : $\quad$ The total length of the backbone grid

$L_{0}: \quad$ The total length of the original grid

$\phi(x)$ : Judging function of connectivity

$\lambda(S)$ : $\quad$ Immigration rate

$\mu(S): \quad$ Emigration rate

$P_{s}: \quad$ Probability of the habitat with $S$ species

$M(S)$ : $\quad$ Variation rate

$M_{\max }$ : Biggest mutation rate 
$P_{m}:$ The maximum of $P_{s}$

$a_{1}$ : Total number of lines.

\section{Conflict of Interests}

The authors have declared that no conflict of interests exists.

\section{Acknowledgments}

This work is funded by the State Grid Corporation of China, Major Projects on Planning and Operation Control of Large Scale Grid (SGCC-MPLG-029-2012), and Natural Science Foundation of China (51207114).

\section{References}

[1] E. Broström, J. Ahlberg, and L. Söder, "Modelling of ice storms and their impact applied to a part of the Swedish transmission network," in Proceedings of the IEEE Lausanne Power Tech Conference, pp. 1593-1598, Lausanne, Switzerland, July 2007.

[2] R. Billinton and G. Singh, "Application of adverse and extreme adverse weather: modelling in transmission and distribution system reliability evaluation," IEE Proceedings: Generation, Transmission and Distribution, vol. 153, no. 1, pp. 115-120, 2006.

[3] R.-A. Hooshmand, R. Hemmati, and M. Parastegari, “Combination of AC transmission expansion planning and reactive power planning in the restructured power system," Energy Conversion and Management, vol. 55, pp. 26-35, 2012.

[4] G. Kjolle, L. Rolfseng, and E. Dahl, "The economic aspect of reliability in distribution system planning," IEEE Transactions on Power Delivery, vol. 5, no. 2, pp. 1153-1157, 1990.

[5] G. Trudel, J.-P. Gingras, and J.-R. Pierre, "Designing a reliable power system: Hydro-Québec's integrated approach," Proceedings of the IEEE, vol. 93, no. 5, pp. 907-917, 2005.

[6] G. Trudel, S. Bernard, and G. Scott, "Hydro-Quebec's defence plan against extreme contingencies," IEEE Transactions on Power Systems, vol. 14, no. 3, pp. 958-966, 1999.

[7] B. A. Hollway and P. G. Neumann, Survivable ComputerCommunication Systems: The Problem Working Group Recommendations, US Army Research Laboratory, Washington, DC, USA, 1993.

[8] R. J. Ellison, D. A. Fisher, R. C. Linger et al., Survivable Network Systems: An Emerging Discipline, Software Engineering Institute, Carnegie Mellon University, Pittsburgh, Pa, USA, 1997.

[9] M. A. Schroeder and K. T. Newport, "Tactical network survivability through connectivity optimization," in Proceedings of the IEEE Military Communications Conference-Crisis Communications: The Promise and Reality (MILCOM '87), pp. 590-597, Washington, DC, USA, October 1987.

[10] A. A. Hagin, "Performability, reliability, and survivability of communication networks: system of methods and models for evaluation," in Proceedings of the 14th International Conference on Distributed Computing Systems, vol. 11, pp. 562-573, June 1994.

[11] A. Dwivedi, X. Yu, and P. Sokolowski, "Identifying vulnerable lines in a power network using complex network theory," in Proceedings of the IEEE International Symposium on Industrial Electronics (ISIE '09), pp. 18-23, Seoul, Republic of Korea, July 2009.
[12] A. Dwivedi, X. Yu, and P. Sokolowski, "Analyzing power network vulnerability with maximum flow based centrality approach," in Proceedings of the 8th IEEE International Conference on Industrial Informatics (INDIN '10), pp. 336-341, Osaka, Japan, July 2010.

[13] W. Yang, T. Bi, S. Huang, A. Xue, and Q. Yang, "An approach for critical lines identification based on the survivability of power grid," Proceedings of the Chinese Society of Electrical Engineering, vol. 31, no. 7, pp. 29-35, 2011.

[14] J. Wang, H. Wang, and G. Zhao, "Index system of quantitative evaluation for information systems survivability," Computer Engineering, vol. 35, no. 3, pp. 54-56, 2009.

[15] A. Soroudi and M. Afrasiab, "Binary PSO-based dynamic multiobjective model for distributed generation planning under uncertainty," IET Renewable Power Generation, vol. 6, no. 2, pp. 67-78, 2012.

[16] R. S. Maciel, M. Rosa, V. Miranda, and A. Padilha-Feltrin, "Multi-objective evolutionary particle swarm optimization in the assessment of the impact of distributed generation," Electric Power Systems Research, vol. 89, pp. 100-108, 2012.

[17] L. Wang and Y. Xu, "An effective hybrid biogeography-based optimization algorithm for parameter estimation of chaotic systems," Expert Systems with Applications, vol. 38, no. 12, pp. 15103-15109, 2011.

[18] M. Ovreiu and D. Simon, "Biogeography-based optimization of neuro-fuzzy system parameters for diagnosis of cardiac disease," in Proceedings of the 12th Annual Genetic and Evolutionary Computation Conference (GECCO '10), pp. 1235-1242, ACM, Portland, Ore, USA, July 2010.

[19] G. Sonakshi, A. Anuja, V. K. Panchal et al., "Extended biogeography based optimization for natural terrain feature classification from satellite remote sensing images," in Proceedings of the International Conference on Contemporary Computing, pp. 262269, IC3, Noida, India, 2011.

[20] D. Simon, "Biogeography-based optimization," IEEE Transactions on Evolutionary Computation, vol. 12, no. 6, pp. 702-713, 2008.

[21] I. Boussaïd, A. Chatterjee, P. Siarry, and M. Ahmed-Nacer, "Hybridizing biogeography-based optimization with differential evolution for optimal power allocation in wireless sensor networks," IEEE Transactions on Vehicular Technology, vol. 60, no. 5, pp. 2347-2353, 2011.

[22] R. Mukherjee and S. Chakraborty, "Selection of the optimal electrochemical machining process parameters using biogeography-based optimization algorithm," The International Journal of Advanced Manufacturing Technology, vol. 64, no. 5-8, pp. 781-791, 2013.

[23] A. Müller, "On the performance of linear adaptive filters driven by the ergodic chaotic logistic map," International Journal of Bifurcation and Chaos in Applied Sciences and Engineering, vol. 22, no. 12, Article ID 1250290, 11 pages, 2012.

[24] P. K. Roy, S. P. Ghoshal, S. S. Thakur et al., "Optimal reactive power dispatch considering flexible AC transmission system devices using biogeography-based optimization," Electric Power Components and Systems, vol. 39, no. 8, pp. 733-750, 2011. 


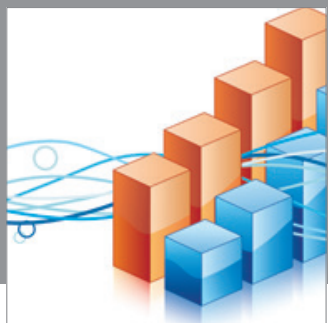

Advances in

Operations Research

mansans

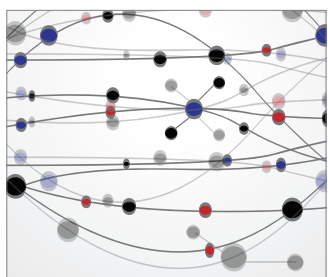

The Scientific World Journal
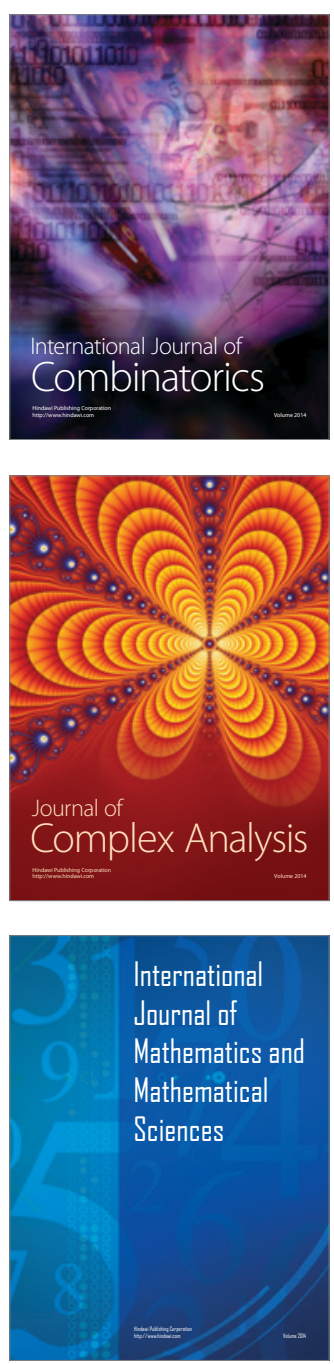
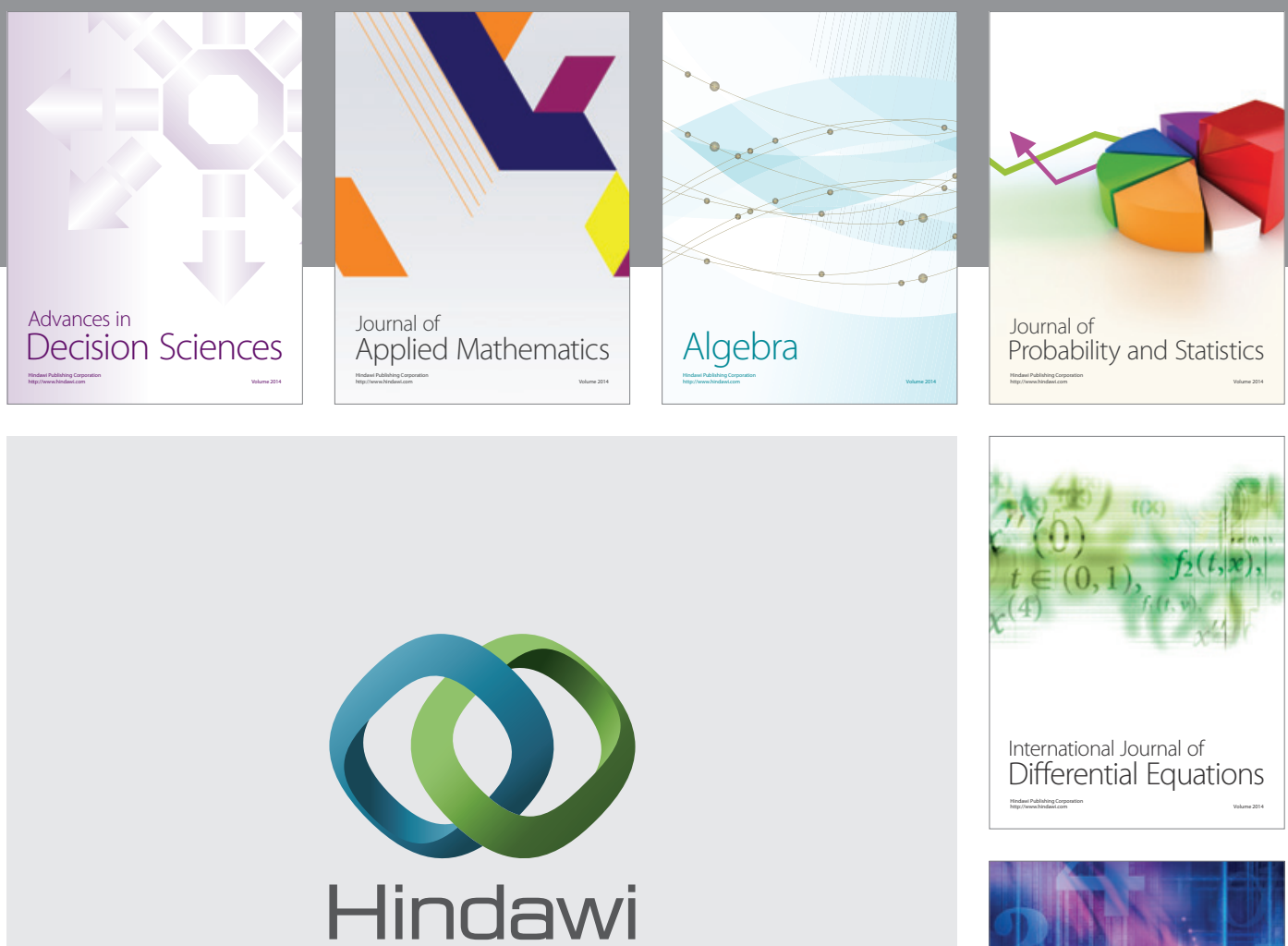

Submit your manuscripts at http://www.hindawi.com
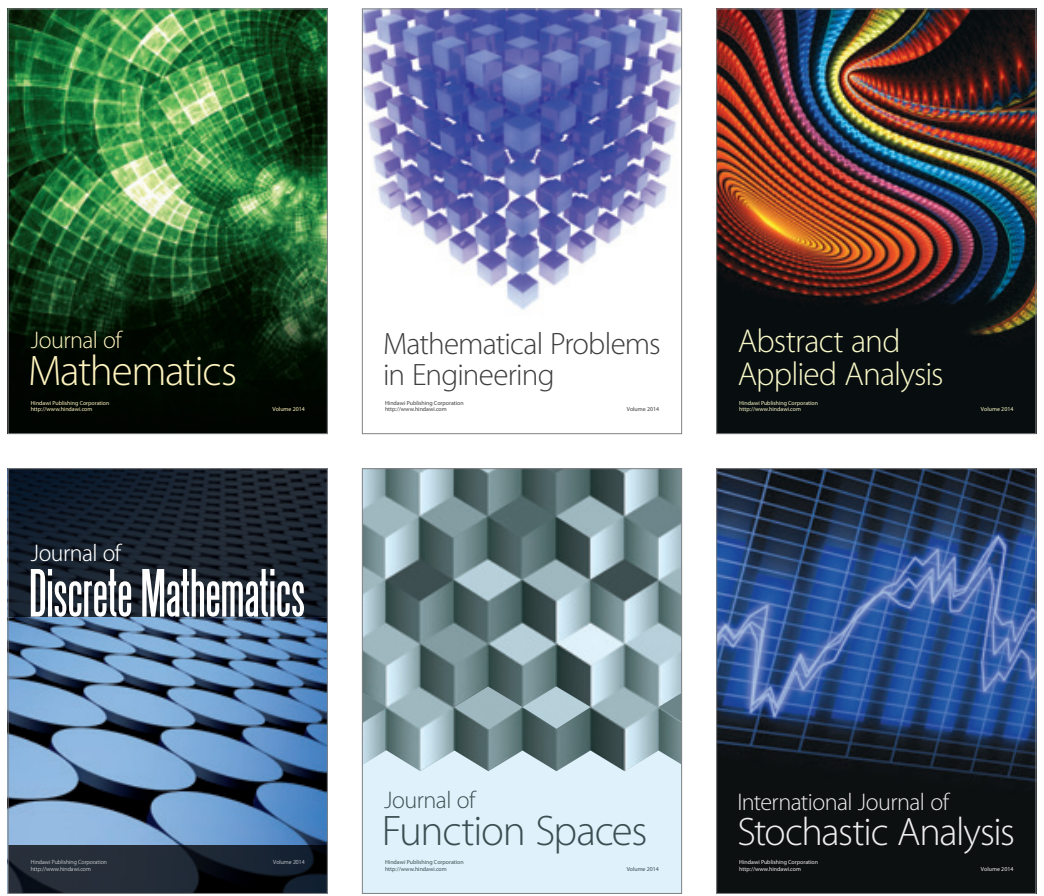

Journal of

Function Spaces

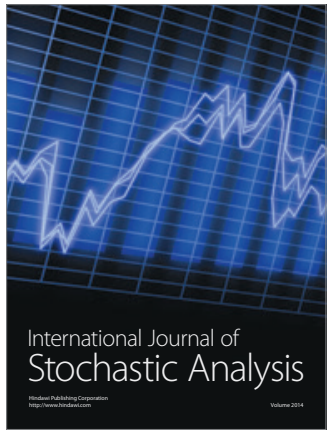

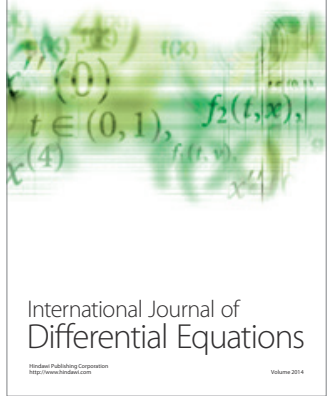
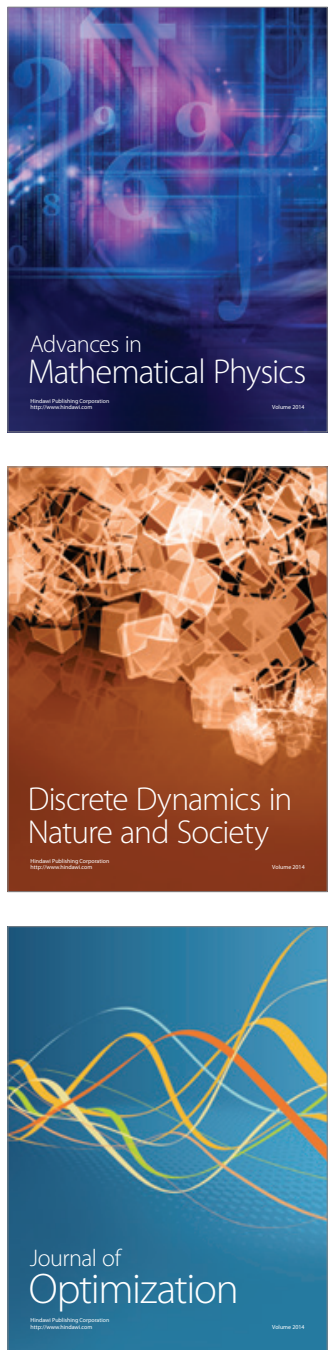